\title{
DESARROLLO DEL CENTRO DE DISPERSIÓN ENTRE LAS PLACAS DE COCO Y NAZCA Y LOS TRAZOS DE LOS PUNTOS CALIENTES
}

\author{
Martin Meschede ${ }^{1}$, Udo Barckhausen²* \& Horst-Ulrich Worm²* \\ 1) Instituto de Geología, Universidad de Tübingen, Sigwartstr. 10, D-72076 Tübingen, Alemania, \\ 2) Instituto de Geofísica, Universidad de Göttingen, Herzberger Landstr. 180, D-37075 \\ Göttingen, Alemania; *ahora: Bundesanstalt für Geowissenschaften und Rohstoffe (Instituto \\ Federal de Geosciencias y Materias Primas), Stilleweg 2, D-30655 Hannover, Alemania.
}

(Recibido 19/4/1999; Aceptado 14/8/1999)

\begin{abstract}
We present a revised plate tectonic model for the evolution of the Cocos-Nazca spreading system. Recently acquired marine magnetic data and existing magnetic, bathymetric, and gravimetric data sets together with new age determinations led to a new interpretation of the complicated situation in the Eastern Panama Basin. According to our model the formation of the southern Cocos plate was governed by spreading at three different ridge axes with alternations between spreading ridges producing a complex magnetic anomaly pattern. In the Cocos and Malpelo ridge area and north of the Grijalva Scarp we have identified two precursors (CNS-1 and CNS-2) of the recently active Cocos-Nacza spreading system (CNS-3). The CNS-1 was active from 22.8 to $19.5 \mathrm{Ma}$, the CNS-2 from 19.5 to $14.7 \mathrm{Ma}$. The spreading direction jumped from NW-SE (CNS-1) to ENE-WSW (CNS-2) at 19.5 Ma and to E-W (CNS-3) at 14.7 Ma. The oceanic crust of the CNS was subsequently thickened and overprinted by hotspot volcanism. The Galapagos hotspot produced the Carnegie ridge on the Nazca plate. A second center of hotspot volcanism about $500 \mathrm{~km}$ away from, but most probably related to the Galapagos hotspot formed the Cocos and Malpelo ridges.
\end{abstract}

\begin{abstract}
RESUMEN: Se presenta un modelo del desarrollo de las placas tectónicas para el centro de dispersión entre las placas Coco y Nazca. Nuevos datos magnéticos marinos y datos existentes de la magnetometría, batimetría y gravimetría; junto con nuevas determinaciones de edades, llevan a una nueva interpretación de la complicada situación del este de la Cuenca de Panamá. En este modelo se interpreta la formación de la parte sur de la placa de Coco como un resultado de un centro de dispersión en tres ejes diferentes, con alternancias entre dorsales de dispersión, lo que produce un trazo complicado de las anomalías magnéticas. En el área de las dorsales de Coco y Malpelo y en el norte del escarpe de Grijalva se han identificado dos precursores (CNS-1 y CNS-2) del centro de dispersión entre las placas Coco y Nazca (CNS-3), actualmente activo. El CNS-1 estuvo activo desde 22,8 hasta 19,5 Ma, el CNS-2 desde 19,5 hasta 14,7 Ma. La dirección de dispersión del CNS-1 cambió de NWSE hacia el ENE-WSW (CNS-2) hace 19,5 Ma y hacia el E-W (CNS-3) hace 14,7 Ma. La corteza oceánica del CNS fue subsecuentemente engrosada y sobrepuesta por el volcanismo de un punto caliente. La dorsal de Carnegie en el norte de la placa de Nazca es un producto del punto caliente de Galápagos. Un segundo centro de volcanismo de un punto caliente, probablemente relacionado al punto caliente de Galápagos, está localizado más de $500 \mathrm{~km}$ al norte de éste y formó las dorsales de Coco y Malpelo.
\end{abstract}

\section{INTRODUCCIÓN}

En el modelo de la evolución tectónica actualmente aceptado, el centro de dispersión entre las placas Coco y Nazca (CNS) inició su evolución hace 25-28 Ma, dividiendo la placa Farallón en las placas Coco y Nazca (Hey, 1977; Lonsdale \& Klitgord, 1978). Actualmente, la corteza oceánica es producida por los centros de dispersión de la dorsal Pacífica del Este (EPR) y del CNS. La parte de la corteza oceánica que estaba formada por el EPR tiene una morfología suave que es normal para las dorsales de dispersión rápida (Hey, 1977; Wilson, 1996), mientras la corteza formada en el CNS evolucionaba en un eje de dispersión lento y por eso muestra una topografía áspera. La parte de la placa del Coco compuesta de corteza formada en el CNS tiene 
un contorno bastante irregular (Fig. 1) que se ha interpretado como el resultado de una dispersión asimétrica en el CNS con dispersión rápida hacia el norte y dispersión lenta hacia el sur. Las anomalías más antiguas de dispersión de la corteza oceánica que se formaban en el CNS existen en la parte más al noreste de la cuenca de Panamá y están correlacionadas a la anomalía 6C (Lonsdale \& Klitgord, 1978). Esta anomalía corresponde a una edad de 23,6 Ma en la escala de edades de polaridades geomagnéticas de Cande \& Kent (1995).

Hey (1977) indica que la historia tectónica del área de Galápagos en el sur del CNS es muy difícil de reconstruir, porque existen dificultades extremas en la correlación de las anomalías antiguas, a pasar de contar con una base de datos bastante buena. Sin embargo, se correlacionan fácilmente las anomalías que tienen una edad más joven que la anomalía 4A. La historia de dispersión de la corteza oceánica de la parte más joven del CNS es presentada por Wilson \& Hey (1995) en un mapa detallado de la parte joven del centro de dispersión incluyendo las anomalías 1 hasta 4A.

Se han interpretado las dorsales asísmicas de Coco y de Malpelo, que se encuentran en la placa de Coco y la dorsal Carnegie en la placa Nazca (Fig. 1) como productos relacionados al punto caliente de Galápagos. Se presume que se formaron cuando el punto caliente de Galápagos inició su actividad hace 20 o $22 \mathrm{Ma}$ (Hey, 1977; Lonsdale \& Klitgord, 1978).

Sin embargo, numerosos datos recientemente obtenidos no se pueden explicar con el modelo aceptado actualmente (von Huene et al., 1995; Mrazek et al., 1996; Kimura et al., 1997; Barckhausen et al., 1998; Meschede et al., 1998).

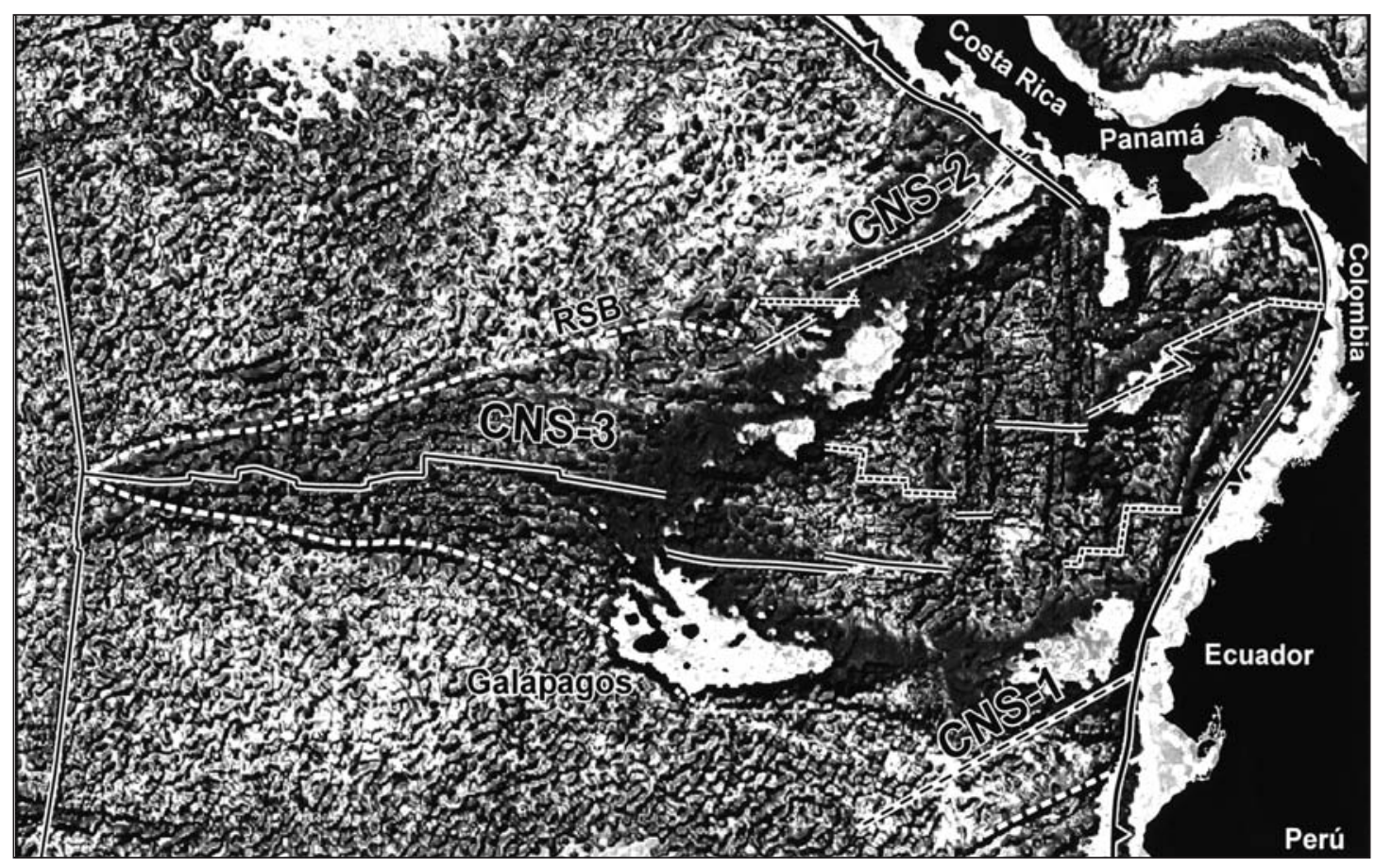

Fig. 1: Modelo sombreado de las colinas ("hillshade model") del centro de dispersión de Coco y Nazca y de la Cuenca este de Panamá (orientación del sol virtual: 323\%11%). Altimetría de los satélites de Smith \& Sandwell (1997). RSB: límite áspero-plano ("rough-smooth boundary"; modificado según Hey, 1977); ejes de dispersión del centro de dispersión Coco y Nazca: CNS-1: 22,8 - 19,6 Ma, CNS-2: 19,6 - 14,7 Ma, CNS-3: 14,7 - Reciente. 
Basándonos en anomalías de la dispersión de la corteza oceánica, datos batimétricos y gravimétricos, nuevos datos para la edad de las rocas oceánicas y condiciones geométricas, presentamos un nuevo modelo de evolución tectónica.

\section{EL MODELO ACTUAL Y LOS DATOS NUEVOS}

La corteza oceánica en el sureste de la península de Nicoya ha sido interpretada como una formación en la EPR (Lonsdale \& Klitgord, 1978; Protti et al., 1994). El límite entre la corteza que se formaba en la EPR y la que se formaba en el CNS, correspondiente al trazo del punto de conección triple entre las placas Pacífica, Coco y Nazca, fue determinado en la esquina sureste de la península de Nicoya, cual fue descrito como el límite entre corteza áspera al norte y plana al sur ("rough-smooth-boundary"; RSB) por Hey (1997). Este autor consideró que la corteza oceánica producida en la EPR era relativamente plana mientras que la corteza oceánica producida en el CNS era áspera (Fig. 1). Datos magnéticos obtenidos durante la investigación del barco científico RV Sonne en 1991/92 (SO-76, von Huene et al., 1995) y 1996 (SO-107, Mrazek et al., 1996) revelaron anomalías magnéticas que sugieren un origen diferente para la corteza oceánica en frente de la península de Nicoya. Barckhausen et al. (1997, 1998) y Meschede et al. (1998) demuestran que las anomalías magnéticas no pueden estar relacionadas a la EPR ni tampoco al CNS, los que actualmente están activos, porque las anomalías de dispersión formadas en la EPR tienen una orientación $\mathrm{N} 15^{\circ} \mathrm{W}$ a $\mathrm{N} 45^{\circ} \mathrm{W}$, mientras que las que se han formado en el actual CNS están orientadas $\mathrm{N} 90^{\circ} \mathrm{E}$. Las anomalías identificadas recientemente en frente de la costa de la península de Nicoya están orientadas $\mathrm{N} 50^{\circ} \mathrm{E}$ y $\mathrm{N} 70^{\circ} \mathrm{E}$. Hey (1977) propone que el CNS ha sufrido rotación desde una orientación primaria de ENE-WSE, a su actual orientación E-W poco después de la formación de las placas Coco y Nazca. Sin embargo, los dos grupos de anomalías de dispersión identificados en frente de la península de Nicoya (Barckhausen et al., 1998) están en contacto discordante, lo que indica un cambio brusco en la dirección de la dispersión. Se observa lo mismo en el contacto entre las anomalías de dirección N70 ${ }^{\circ}$ de Barckhausen et al. (1998) y las anomalías con la dirección E-W de Hey (1977).

Las dorsales de Coco y de Malpelo se han interpretado como un trazo originalmente contínuo, formado en el punto caliente de Galápagos (Hey, 1977; Johnston \& Thorkelson, 1997; Sinton et al., 1997) y que fue separado posteriormente por la zona de fractura de Panamá. Sin embargo, el archipiélago de Galápagos, con su volcanismo de punto caliente (Global volcanism report, 1995), está localizado $250 \mathrm{~km}$ al sur del eje del CNS y así se excluye la formación de una traza de un punto caliente al norte del eje de dispersión. Por eso, se han asumido múltiples saltos del eje del CNS hacia el norte, para explicar el origen de la dorsal de Coco en el punto caliente de Galápagos (Hey, 1977; Wilson \& Hey, 1995). Datos altimétricos de satélites (Smith \& Sandwell, 1997) indican, que la dorsal de Coco termina alrededor de $200 \mathrm{~km}$ al norte del eje del CNS (Fig. 1). Si se asume, que la dorsal fue formada en el punto caliente de los Galápagos, entonces esta dorsal debería ser prominente cerca del eje de dispersión.

Nuevos datos batimétricos obtenidos fuera de la península de Osa (en el sur de Costa Rica) durante la investigación del barco científico RV Sonne SO-107 (Mrazek et al., 1996), el mapa batimétrico de Lonsdale \& Klitgord (1978) y los datos altimétricos de satélites (Smith \& Sandwell, 1997) indican la existencia de un graben tectónico, cuyo rumbo es ENE-WSW tanto en la cresta y en el flanco noroeste de la dorsal de Coco, como en la cresta de la dorsal de Malpelo (Fig. 1). Estas estructuras, cuya dirección difiere del rumbo de las dorsales, no son claramente relacionadas a un punto caliente, por lo tanto requieren otra explicación.

Hey (1977), Hey et al. (1978) y Lonsdale $\&$ Klitgord (1978) han mencionado que la disposición de las anomalías más viejas que la anomalía 4A son muy difíciles de determinar. Las anomalías jóvenes del centro de dispersión entre las placas Coco y Nazca están bien establecidas (Wilson \& Hey, 1995) y, por eso la historia de este 
centro de dispersión está bien definida para el tiempo de la anomalía 4A (9,6 Ma según la escala de tiempos de polaridades geomagnéticos de Cande \& Kent, 1995) hasta el Reciente. Sin embargo, las anomalías más viejas en la placa de Coco no son suficientemente explicadas con el modelo actual.

\section{CONDICIONES GEOMÉTRICAS}

Se han revisado todos los datos tomados por los barcos científicos y las informaciones batimétricas (Lonsdale \& Klitgord, 1978, von Huene et al., 1995; National Geophysical Data Center, 1996; Mrazek et al., 1996, Smith \& Sandwell, 1997), que existen en el área de la cuenca de Panamá Este y en la zona de dispersión Coco y Nazca. Las anomalías magnéticas están orientadas de NE al SW y de ENE al WSW en las dorsales de Coco y de Malpelo y, ambas están en contacto discordante y claramente diferentes de las anomalías de la zona de dispersión actual de
Coco y Nazca y de las anomalías orientadas de NW-SE en la EPR (Fig. 2a).

Se han identificado dos grupos de anomalías magnéticas en los perfiles que cortan las dorsales de Coco y Malpelo: (1) un grupo más viejo con dirección $\mathrm{N} 50^{\circ} \mathrm{E}$ y (2) un grupo más joven con dirección $\mathrm{N} 70^{\circ} \mathrm{E}$ (Fig. 2a). Las anomalías simétricas del grupo más joven indican que el centro de la zona de dispersión abandonada se encontraba en el flanco noroeste de la dorsal de Coco y en la cresta de la dorsal de Malpelo (Fig. 3). Se presume que el centro del sistema de dispersión más viejo está en el sur de la dorsal de Carnegie (Fig. 4). Ambos sistemas de dispersión abandonados, son los precursores del centro de dispersión Coco y Nazca, que está activo en el presente (CNS-3) y, por eso se han denominado CNS-1 y CNS-2. La identificación de estas anomalías se hizo con base en un perfil sintético (Cande \& Kent, 1995) y se pueden reconocer las anomalías $6 \mathrm{Bn} .1 \mathrm{n}$ hasta $6 \mathrm{An} .2 \mathrm{r}$ para el sistema de dispersión más viejo (CNS-1) y, las anomalías 6 hasta 5ADr para el sistema de dispersión más

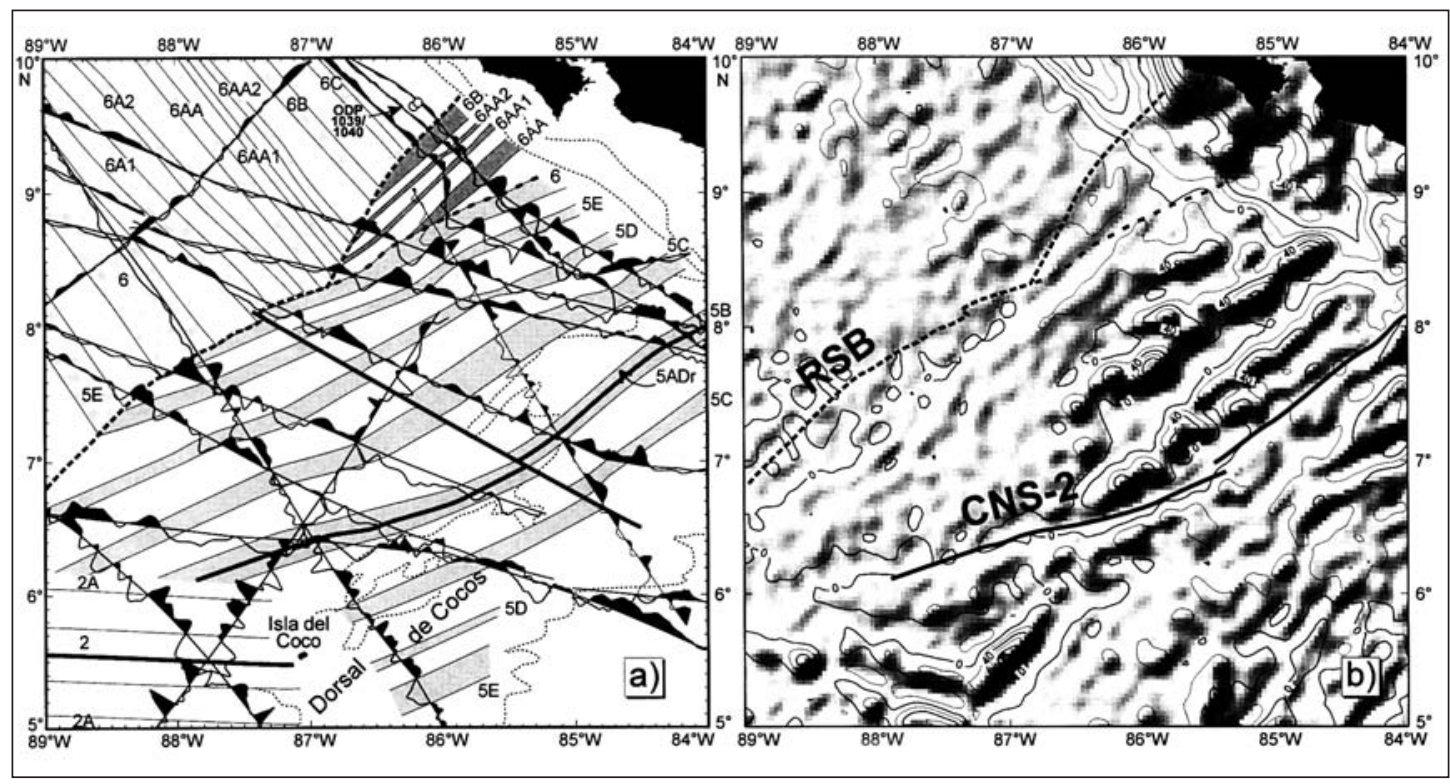

Fig. 2: a) Las anomalías magnéticas en la región de la dorsal de Coco. Correlación de los trazos de los barcos representativos y la identificación de las isocronas referidas en la Fig. 3. Los trazos de los barcos provienen del National Geophysical Data Center (1996), de von Huene et al. (1995), y de Mrazek et al. (1996). Las anomalías 6 y 6A en la corteza oceánica fueron formadas en la EPR (esquina superior izquierda) según Wilson (1996). b) Mapa de las anomalías gravimétricas aire libre de la misma región mostrado en la Fig. 2a. Los datos gravimétricos son de Sandwell \& Smith (1997). 
joven (CNS-2). Se determinaron velocidades de dispersión de $50 \mathrm{~mm} / \mathrm{a}$ para el CNS-1 y de 40 $\mathrm{mm} / \mathrm{a}$ para el CNS-2. Muy probablemente las anomalías ausentes 6A2 y 6A1 están representadas en la parte sur de la zona de dispersión abandonada, que fue transferida a la placa de Nazca, en el sur de la zona de dispersión actualmente activa CNS-3 (Fig. 4).

La identificación de las anomalías más viejas del CNS en la región sur ha sido recientemente revisada por Barckhausen et al. (en prep.) y coincide con la interpretación presentada en la Fig. 4. Se han reexaminado las anomalías magnéticas en el norte del escarpe de Grijalva usando otros perfiles que fueron obtenidos por Hey (1977) y Lonsdale \& Klitgord (1978), lo que confirma los resultados de Hey (1977) y Lonsdale \& Klitgord (1978). La anomalía más vieja en el flanco norte del escarpe de Grijalva es 6B1 (22,8 Ma según la escala del tiempo de reversiones magnéticos de Cande \& Kent (1995) y se pueden identificar las anomalías subsecuentes hasta 6A1 (20,6 Ma; Fig. 5).

Fuertes anomalías magnéticas lineales y simétricas en el oeste de la Isla del Coco indican un segundo eje de una zona de dispersión pequeña con dirección E-W, que estaba activa desde

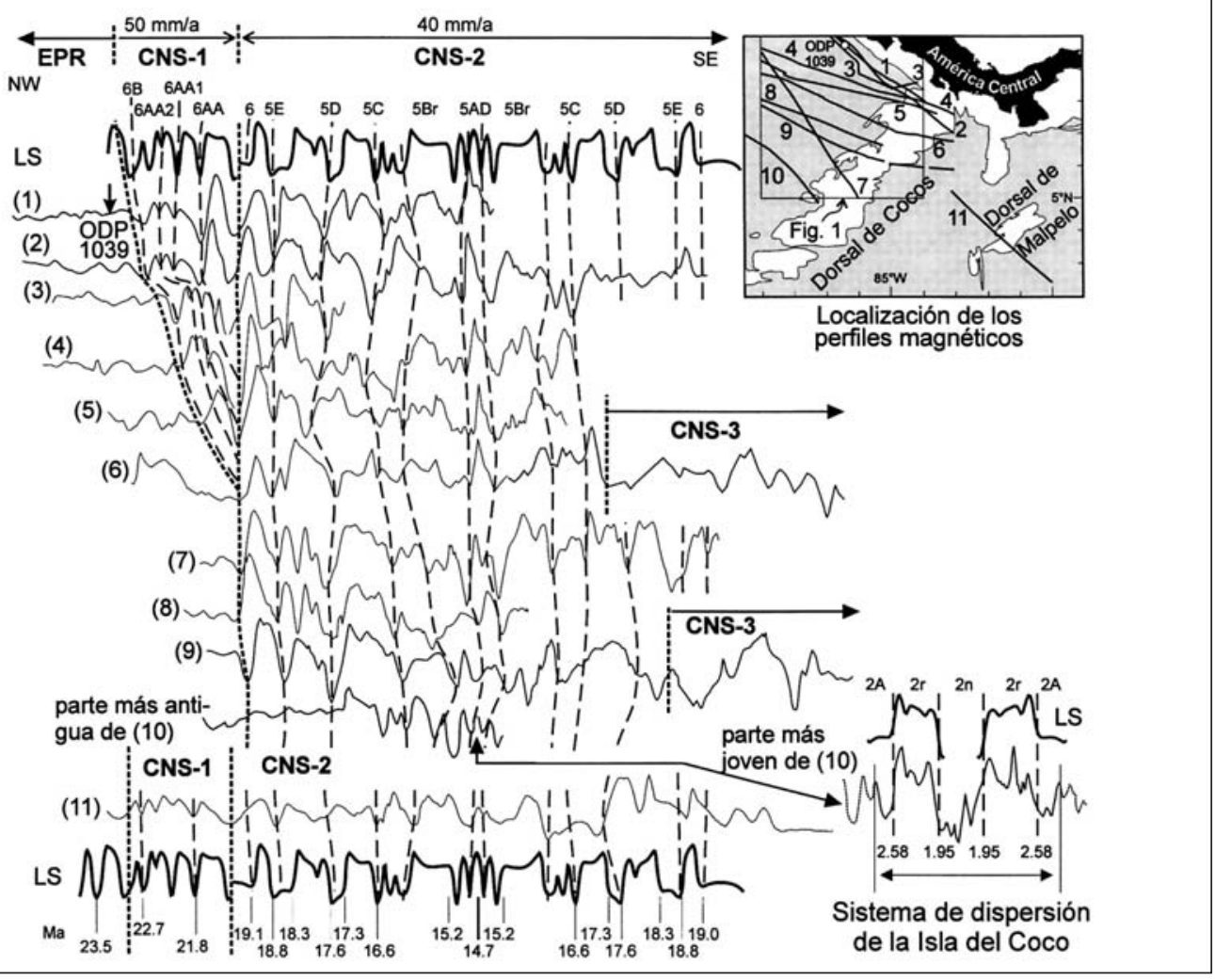

Fig. 3: Correlación de los perfiles magnéticos en las dorsales de Coco y Malpelo. La línea 1 intercepta el sitio 1039 de ODP. La línea 7 es un ejemplo de un perfil magnético simétrico que corta el centro de dispersión extinto del precursor 2 del sistema de dispersión de Coco y Nazca (5ADr). LS: Línea sintética de anomalías magnéticas. EPR: corteza oceánica formada en la dorsal del Pacífico este. CNS-1: corteza oceánica formada en el precursor 1 del centro de dispersión de Coco y Nazca (22,8 - 19,5 Ma). CNS-2: corteza oceánica formada en el precursor 2 del centro de dispersión de Coco y Nazca (19,5 - 14,7 Ma). CNS-3: corteza oceánica formada en el centro de dispersión actual de Coco y Nazca (desde 14,7 Ma). La identificación de las isocronas se refieren a la escala de tiempos de las polaridades geomagnéticas (Cande \& Kent, 1995). En el mapa de ubicación se indican las trayectorias de los barcos. 
3,0 hasta 1,8 Ma (anomalías 2A hasta 2; Figs. 2a, $3,4)$, y que son paralelas a la zona de dispersión acualmente activa de Coco y Nazca. Esta zona de dispersión abandonada probablemente está relacionada a otro centro extinto del sistema Coco y Nazca, identificado por Anderson et al. (1976) y Batiza (1986) (Fig. 4).

Las edades de las anomalías magnéticas están de acuerdo con los datos de los sitios 1039 y 1040 del ODP Leg 170 (Ocean Drilling Program). Se ha determinado la edad magnetoestratigráfica de 16,5 Ma en sedimentos directamente sobreyacientes a un sill gabróico en la placa de Coco. Perfiles sísmicos indican que las capas sedimentarias continúan bajo del sill gabróico, lo que implica una edad de la corteza oceánica entre 17 y 23 Ma (Kimura et al., 1997). Según los datos magnéticos, la corteza oceánica en el oeste de la península de Nicoya es parte de la corteza oceánica formada en la EPR (Barckhausen et al., en prep.). En de los sitios 1039 y 1040 de ODP Leg 170 la corteza es más vieja que la anomalía 6C, que indica una edad de alrededor de $23 \mathrm{Ma}$. Esto coincide con la edad estimada para la corteza oceánica derivada de los datos de las perforaciones (Kimura et al., 1997).

Se relacionan las anomalías más viejas que la anomalía 6B, al sistema de dispersión

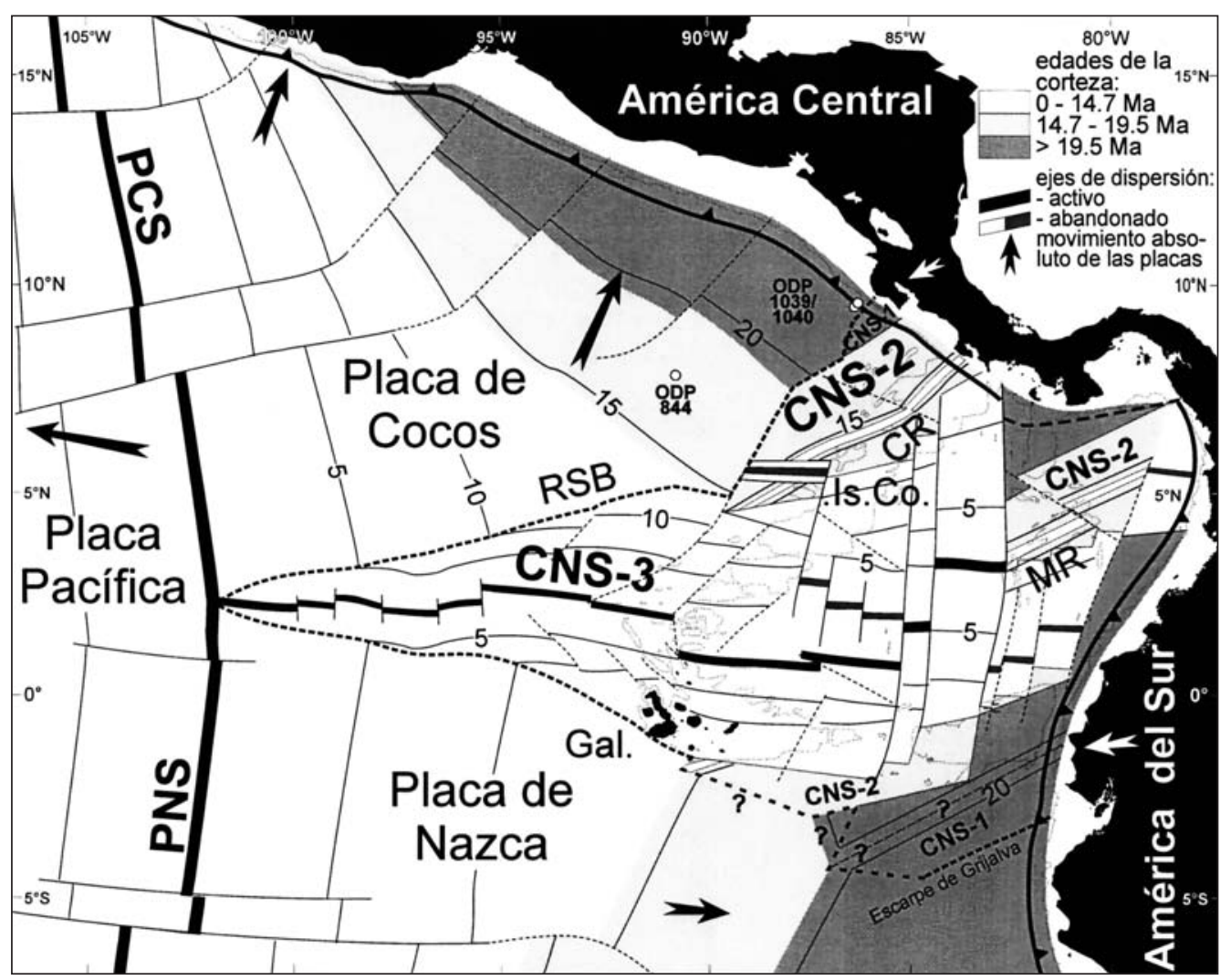

Fig. 4: Mapa tectónico de las placas Coco y del norte de Nazca. CNS-1, CNS-2: Precursores 1 y 2 del sistema de dispersión de Coco y Nazca. CNS-3: sistema de dispersión actual de Coco y Nazca. CR: Dorsal de Coco. Gal.: Archipiélago de Galápagos. MR: Dorsal de Malpelo. PCS: Sistema de dispersión del Pacífico y Coco. PNS: Sistema de dispersión del Pacífico y Nazca. RSB: Límite áspero-plano ("rough smooth boundary"; modificado según Hey, 1977). Alineación del CNS-3 según Searle (1989) y Wilson \& Hey (1995). Movimiento absoluto de las placas según De Mets (1990). 
viejo entre las placas Pacífica y Farallón. La anomalía más vieja preservada del sistema de dispersión CNS-1 (6B, Fig. 3) intersecta la misma anomalía en la corteza formada en la EPR, alrededor de $80 \mathrm{~km}$ al suroeste de los sitios 1039/1040 del ODP (Fig. 2a). La determinación de las anomalías en la corteza formada en la EPR se derivan de los datos de las edades del ODP Leg 138 en el sitio 844 (Fig. 4; Mayer et al., 1992) que está localizado entre la anomalía 5D. Wilson (1996) ha extrapolado las anomalías formadas en la EPR hacia la costa hasta la anomalía 6A; Barckhausen et al. (subm.) continúan la extrapolación hasta la fosa Centroamericana (Fig. 2a). Las anomalías que antes estaban asociadas con la parte sureste del sistema de dispersión CNS-1 ahora están desplazadas hacia el sur, por la zona de dispersión activa CNS-3 y están localizadas actualmente en la placa de Nazca, al norte del escarpe de Grijalva.

De los datos altimétricos de satélites (Smith \& Sandwell, 1997; Fig. 1) se han obtenido en las dorsales de Coco y de Malpelo estructuras de graben con un rumbo ENE-WSW, que coinciden con la dirección del eje del sistema de dispersión abandonado CNS-2. Actualmente el eje abandonado del CNS-2 está localizado directamente en frente de la península de Osa, donde se ha encontrado una estructura de un graben por investigaciones batimétricas con "hydrosweep", frente a la costa Pacífica de Costa Rica (Mrazek et al., 1996).

El mapa de anomalías gravimétricas aire libre del área de estudio (Sandwell \& Smith, 1997) indica ambos rasgos, lineamientos paralelos a la dorsal de Coco y lineamientos paralelos a las anomalías magnéticas (Fig. 2b). Una disminución de la gravedad elongada coincide con el eje abandonado del CNS-2 al oeste del grado de longitud $86^{\circ} \mathrm{W}$. La disminución de la gravedad es típica para los ejes de dispersión abandonados y esto confirma la interpretación de las anomalías magnéticas.

Se ha denominado el límite entre la corteza oceánica formada en la EPR y la corteza formada en el CNS como "límite áspero-plano" ("rough-smooth boundary"; Hey, 1977). Este límite tiene un contorno irregular con dos inflexiones importantes: Una al norte, alrededor de $5^{\circ} \mathrm{N}, 89,5^{\circ} \mathrm{W}$ - donde el límite cambia de un rumbo de ENE-WSW, a un rumbo NE-SW; y otra en el sur, alrededor de $4,5^{\circ} \mathrm{S}, 84,5^{\circ} \mathrm{W}$ - donde el rumbo cambia de E-W a NE-SW. Para explicar esta geometría irregular, el modelo anterior asume una dispersión bastante asimétrica en la zona de dispersión de Coco y Nazca. En cambio, se concluye que esta geometría es el resultado de múltiples saltos de la dirección de los ejes de la dispersión y de la evolución de diferentes sistemas subsecuentes de dispersión (Fig. 5).

Con los dos sistemas de dispersión abandonados, CNS-1 y CNS-2, no se puede explicar la corteza oceánica engrosada de las dorsales de Coco y Malpelo. Ambas dorsales consisten de corteza oceánica ligera de un espesor de por lo menos $15 \mathrm{~km}$ (Astorga et al., 1989). Se toma la orientación de la dorsal de Coco, señalando hacia el archipiélago de Galápagos, y las características isotópicas de las muestras de la dorsal de Coco como evidencia para su formación en el punto caliente de Galápagos. Sin embargo, la relación directa entre el punto caliente de Galápagos y las dorsales es geométricamente difícil de explicar, porque el punto caliente de Galápagos está localizada más de $250 \mathrm{~km}$ al sur de la zona de dispersión activa CNS-3. Wilson \& Hey (1995) han concluido que el punto caliente estaba localizado bajo la placa Nazca, por lo menos durante los últimos 5 Ma.

Si el punto caliente de Galápagos estaba localizado en el centro del eje de dispersión CNS-2 cuando este eje estaba activo, entonces la dorsal de Coco, considerándola como un trazo de este punto caliente, se hubiero formado al norte del eje de dispersión CNS-2. Sin embargo, la dorsal de Coco está localizada al sur de este eje y su orientación difiere $8^{\circ}$ de su rumbo (Fig. 4). Por eso, se presume la existencia de un segundo centro de actividad de un punto caliente localizado alrededor de $500 \mathrm{~km}$ al noroeste de Galápagos que ha sobrepuesto los sistemas de dispersión después del cese de la dispersión y que es responsable del engrosamiento de la corteza de las dorsales de Coco y Malpelo. La geometría de la dorsal de Coco implica que este segundo centro de actividad estaría localizado en el área al suroeste de la isla del Coco (Fig. 4), donde se observa un 
volcanismo alcalino del Plioceno (Castillo et al., 1988). Se presume, que la isla del Coco representa la parte de la dorsal de Coco que atravesaba el punto caliente durante el Plioceno. Un cambio del centro de actividad volcánica en la isla del Coco de noreste hacia suroeste esta documentado (Castillo et al., 1988) y es consistente con el modelo que se presenta en este trabajo. Las características geoquímicas de las rocas volcánicas de la isla del Coco son similares á las de Galápagos, que representan un volcanismo típico de los puntos calientes. Por eso, se presume una relación del centro de actividad de un punto caliente en el norte con la del punto caliente de Galápagos.

La reconstrucción paleogeográfica del CNS-2 hace 14,7 Ma, coloca las dorsales de Coco

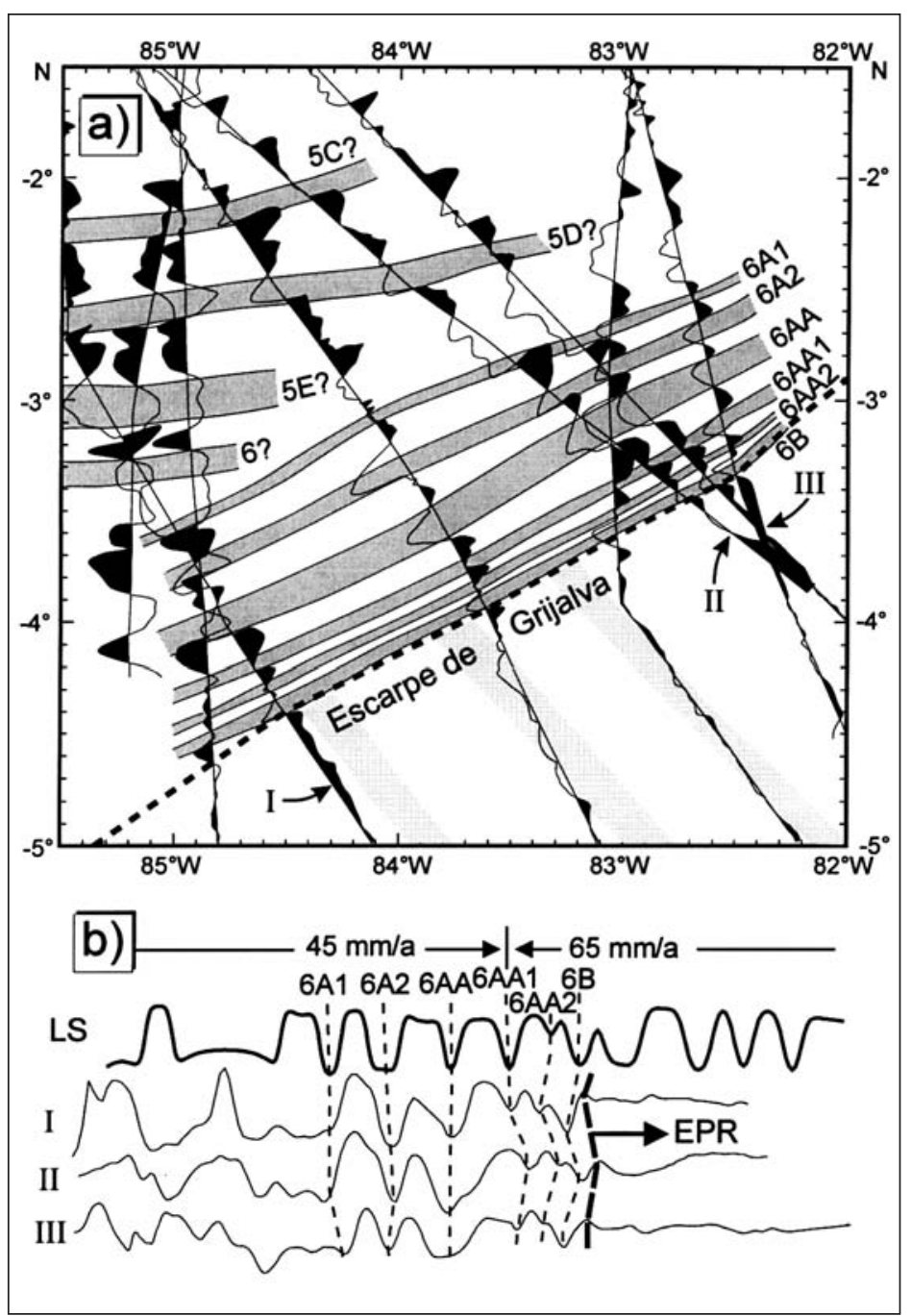

Fig. 5: a) Anomalías magnéticas en el área alrededor del escarpe de Grijalva en la placa de Nazca. Los trazos de los barcos provienen del National Geophysical Data Center (1996). El escarpe de Grijalva es paralelo a las anomalías magnéticas del CNS-1 en el lado NW del escarpe indicando que la formación de esta parte de la litósfera ocurrió en un límite de placas paralelo y no en un punto triple; b) correlación de tres perfiles magnéticos (I, II, III) marcado en el mapa con una línea sintética propuesta para la identificación de las anomalías magnéticas. LS: Línea sintética de las anomalías magnéticas. EPR: Corteza oceánica formada en la dorsal del Pacífico Este ("East Pacific Rise"). 
y Malpelo en forma alineada (Fig. 5; Hey, 1977; Lonsdale \& Klitgord, 1978). La parte ausente del sistema de dorsales Coco y Malpelo de 200-250 km, fue subducido bajo del arco volcánico Centroamericano y es responsable del levantamiento de la cordillera de Talamanca, en la parte central y sur de Costa Rica. En esta área las rocas plutónicas del Mioceno afloran a alturas mayores de $3500 \mathrm{~m}$ sobre el nivel del mar (Drummond et al., 1995; de Boer et al., 1995, Graefe, 1998). Asumiendo un período de subducción constante de $81 \mathrm{~mm} / \mathrm{a}$ (DeMets et al., 1990), el comienzo de la subducción de la dorsal de Coco ocurrió hace aproximadamente 3-4 Ma. Lo cual está apoyado por los datos geológicos de Costa Rica, que documentan un cambio de la sedimentación marina hacia continental (Sprechmann et al., 1994), mostrando una somerización desde condiciones batiales hasta cercanas a la costa en la región de tras-arco de Costa Rica y la parte oeste de Panamá (Collins, 1993; Collins et al., 1995). La ausencia de estratovolcanes en la parte sureste de Costa Rica desde el Plioceno (De Boer et al., 1995) también apoya este modelo.

\section{CONCLUSIONES}

Usando los análisis de las anomalías magnéticas, la interpretación de los datos batimétricos y gravimétricos y los nuevos datos de control de edad se propone para la evolución tectónica de la placa de Coco un modelo de múltiples etapas (Fig. 6). Considerando que el material de los trazos de un punto caliente es más joven que la corteza oceánica de la placa subducida, se concluye que el centro de dispersión de Coco y Nazca evolucionaba independientemente de la actividad del punto caliente de Galápagos. Las placas de Coco y Nazca, por eso, estaban sobrepuestos por los trazos de un punto caliente.

La placa Farallón se separó en las placas de Coco y Nazca, hace 22,8 Ma, cuando el primer precursor del sistema de dispersión Coco y Nazca (CNS-1) iniciaba su actividad. Se pueden encontrar restos de este sistema de dispersión en la placa de Coco, frente a la península de Nicoya y, también probablemente al norte del escarpe de Grijalva en la placa de Nazca. La edad de formación de las placas de Coco y Nazca está documentada por las anomalías magnéticas que se pueden determinar como anomalía 6B. El sistema CNS-1, orientado $\mathrm{N} 50^{\circ} \mathrm{E}$, estuvo activo desde 22,8 hasta 19,5 Ma (anomalías 6B

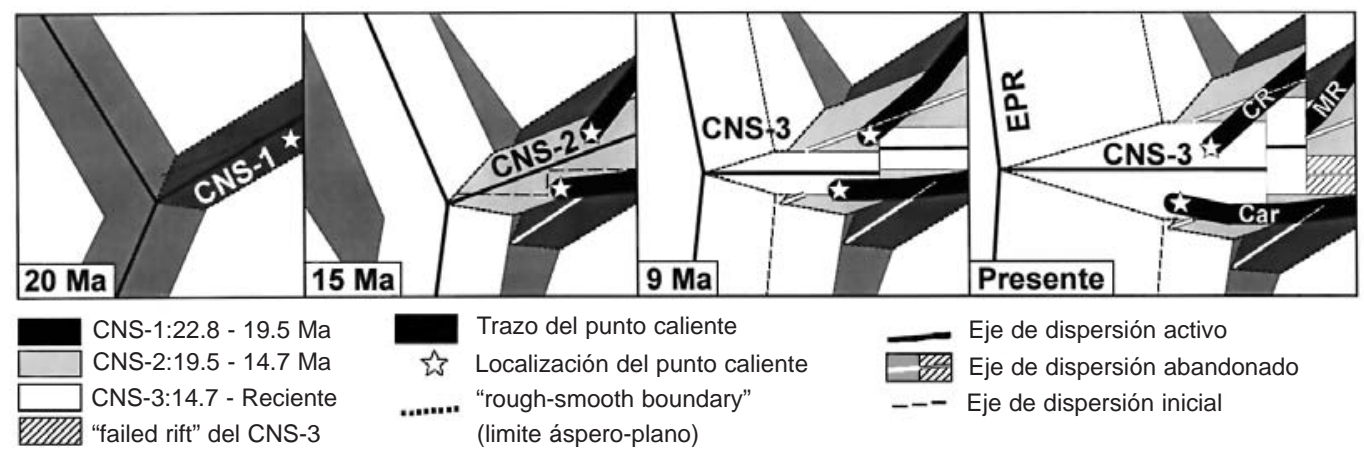

Fig. 6: Esquema simplificado de la evolución del sistema de dispersión de Coco y Nazca y sus precursores. CNS-1: Sistema de dispersión de Coco y Nazca 22,8 - 19,5 Ma. CNS-2: Sistema de dispersión de Coco y Nazca 19,5 - 14,7 Ma. CNS-3: Sistema de dispersión de Coco y Nazca 14,7 Ma - Presente. Car: Dorsal de Carnegie. CR: Dorsal de Coco. EPR: Dorsal del Pacífico Este. MR: Dorsal de Malpelo. 
hasta 5ADr). Seguido por el sistema más joven CNS-2, orientado $\mathrm{N} 70^{\circ} \mathrm{E}$, que estuvo activo desde 19,5 hasta 14,7 Ma. El sistema de dispersión de Coco y Nazca, que se encuentra actualmente activo y orientado E-W, se originó hace 14,7 Ma, cuando la dirección de la dispersión cambió de un rumbo ENE-WSW a E-W. Algunas dorsales extintas indican también los cambios de los ejes de dispersión más jóvenes.

La dorsal de Malpelo y la mayoría de la dorsal de Coco se formaron en un centro de actividad de un punto caliente, que se propone estuvo localizado alrededor de $200 \mathrm{~km}$ al suroeste de la isla del Coco. La dorsal de Carnegie, en la placa de Nazca, es un producto de la actividad en el punto caliente de Galápagos, mientras que las condiciones geométricas excluyen una relación directa entre la dorsal de Malpelo y de la parte mas jóven de la dorsal de Coco, y el punto caliente de Galápagos, actualmente activo. Las determinaciones de las edades de las muestras en la dorsal de Coco frente a Costa Rica (Werner et al., 1999), indican que la formación del trazo del punto caliente fue después de la extinción del CNS-2. Antes de la formación de la zona de fractura de Panamá las dorsales de Coco y Malpelo formaban un trazo continuo de un punto caliente. La parte ausente, de 200-250 km, de la dorsal que une las dos dorsales en las reconstrucciones paleogeográficas fue subducido desde el Plioceno. Esta subducción produjo la exhumación del istmo de América Central durante los últimos 3,5-4 Ma.

\section{AGRADECIMIENTOS}

Se agradece a todos los colegas que contribuyeron al desarrollo de estas nuevas ideas. En particular a Hugh Cowan (Lower Hutt, Nueva Zelandia), Ernst Flueh (Geomar Kiel, Alemania), Wolfgang Frisch (Tuebingen, Alemania), Marc Gutscher (Montpellier, Francia), David Naar (Univ. S-Florida, FL, EE.UU.), David Scholl (Menlo Park, EE.UU.), Eli Silver (Santa Cruz, EE.UU), Christopher Sinton (Alfred, NY, EE.UU), and Roland von Huene (Geomar Kiel, Alemania). Balász Székely (Tuebingen, Alemania) ayudó preparando los datos altimétricos de satélites y Ana Leyla Chinchilla (Tuebingen, Alemania) corrigió el texto español. La fundación Alemana de Ciencias (DFG) ha dado el apoyo económico.

\section{REFERENCIAS}

ANDERSON, R.N., MOORE, G.F., SCHILT, S.S., CARDWELL, R.C., TREHU, A. \& VACQUIER, V., 1976: Heat flow near a fossil ridge on the north flank of the Galapagos spreading center. - J. Geophys. Res. 81: 1828-1838.

ASTORGA, A., FERNÁNDEZ, J.A., BARBOZA, G., CAMPOS, L., OBANDO, J., AGUILAR, A. \& OBANDO, L.G., 1989: Cuencas sedimentarias de Costa Rica: Evolución Cretácico Superior - Cenozoica y potencial de hidrocarburos. - Symp. of Energy and Mineral Potentials, Central American - Caribbean Region, San José, Costa Rica, Circum Pacific Council, 23 págs.

BARCKHAUSEN, U., ROESER, H.A. \& VON HUENE, R., 1997: New insight into the structure of the Cocos plate offshore Costa Rica from seamagnetic measurement. Zentralbl. Geol. Pal., parte 1, 385-391.

BARCKHAUSEN, U., ROESER, H.A. \& VON HUENE, R., 1998: Magnetic signature of upper plate structures and subducting seamounts at the convergent margin off Costa Rica. -J. Geophys. Res. 103: 7079-7093.

BARCKHAUSEN, U., RANERO, C., VON HUENE R. \& MESCHEDE, M. (en prep.): Revised tectonic boundaries in the Cocos Plate off Costa Rica and analogous segmentation of the continent. - sometido a Tectonics.

BATIZA, R., 1986: Failed rifts. - En: E.L. WINTERER, D.M. HUSSONG \& R.W. DECKER (eds.): The Eastern Pacific Ocean and Hawaii. - The Geology of North America, 
Vol. N, Cap. 11: 177-186.

CANDE, S.C. \& KENT, D.V.J., 1995: Geomagnetic polarity time scale. - J. Geophys. Res. 100(B4): 6093-6095.

CASTILLO, P., BATIZA, R., VANKO, D., MALAVASSI, E., BARQUERO, J. \& FERNANDEZ, E., 1988: Anomalously young volcanoes on old hot-spot traces: I. Geology and petrology of Cocos Island. Geol. Soc. Am., Bull. 100: 1400-1414.

\section{CIRCUM-PACIFIC COUNCIL FOR ENERGY} AND MINERAL RESOURCES, 1981: Plate-tectonic map of the Circum-Pacific region, southeast quadrant, $1: 10000000$. - Am. Ass. Petrol. Geol., Tulsa Oklahoma.

COLLINS, L.S., 1993: Neogene paleoenvironments of the Bocas del Toro Basin, Panama. - J. Paleont. 67: 699-710.

COLLINS, L.S., COATES, A.G., JACKSON, J.B.C. \& OBANDO, J., 1995: Timing and rates of emergence of the Limon and Bocas del Toro basins: Caribbean effects of Cocos ridge subduction?. -En: P. MANN (ed.): Geologic and Tectonic Development of the Caribbean plate boundary in southern Central America. - Geol. Soc. Am., Spec. Pap. 295: 263-289.

DeBOER, J.Z., DRUMMOND, M.S., BORDELON, M.J., DEFANT, M.J., BELLON, H. \& MAURY, R.C., 1995: Cenozoic magmatic phases of the Costa Rican island arc (Cordillera de Talamanca). - Geol. Soc. Am., Spec. Pap. 295: 35-55.

DeMETS, C., GORDON, R.G., ARGUS, D.F. \& STEIN, S., 1990: Current plate motions. Geophys. J. Int. 101: 425-478.

DRUMMOND, M.S., BORDELON, M., DE BOER, J., DEFANT, M.J., BELLON \& FEIGENSON, M.D., 1995: Igneous petrogenesis and tectonic setting of plutonic and volcanic rocks of the Cordillera de Talamanca, Costa Rica-Panama, Central American arc. - Am. J. Sci. 295: 875-919.

GLOBAL VOLCANISM REPORT, 1995: Smithsonian Inst., Washington, DC: 20(1):2-3.

GRAEFE, K., 1998: Exhumation and thermal evolution of the Cordillera de Talamanca (Costa Rica: Constraints from fission track analysis, 40Ar-39Ar, and 87Rb-87Sr chronolgy. - Tüb. Geowiss. Arb. 39, 113 págs.

HEY, R., 1977: Tectonic evolution of the CocosNazca spreading center. -Geol. Soc. Am., Bull. 88: 1404-1420.

HEY, R., JOHNSON, G.L. \& LOWRIE, A., 1978: Recent plate motions in the Galapagos area. center. - Geol. Soc. Am.,Bull. 88: 1385-1403.

JOHNSTON, S.T. \& THORKELSON, D.J., 1997: Cocos-Nazca slab window beneath Central America. - Earth Planet. Sci. Lett. 146: 465-474.

KIMURA, G., SILVER, E., BLUM, P., et al., 1997: Initial Report, Proceedings of the Ocean Drilling Program, 170.

LONSDALE, P. \& KLITGORD, K.D., 1978: Structure and tectonic history of the eastern Panama Basin, center. - Geol. Soc. Am.,Bull. 89: 981-999.

MAYER, L., PISIAS, N. et al., 1992: Initial Report, Proc. Ocean Drill. Progr., 138.

MESCHEDE, M., BARCKHAUSEN, U. \& WORM, H.-U., 1998: Extinct spreading ridges on the Cocos Plate. - Terra Nova, 10: 211-216.

MRAZEK, J. SPANGENBERG, T. \& VON HUENE, R., 1996: Geologische und Geophysikalische Untersuchungen vor Costa Rica und Nicaragua - Beiträge zum Verständnis des akti- 
ven ostpazifischen Kontinentalrandes. - FS Sonne Fahrtbericht SO 107, Pacomar 3, Balboa/Panama - San Francisco/USA, 10.03. - 14.04.1996, Unpubl. report, Geomar, Kiel, Ernst-Moritz-Arndt Universität Greifswald, 172 págs.

NATIONAL GEOPHYSICAL DATA CENTER, 1996: Marine Geophysical DATA, CDROM.

PROTTI, M., GUENDEL, F. \& McNALLY, K., 1994: The geometry of the Wadati-Benioff zone under southern Central America and its tectonic significance: results from a high-resolution local seismographic network. - Physics of the Earth and Planetary Interior, 84: 271-287.

SANDWELL, D.T. \& SMITH, W.H.F., 1997: Marine gravity anomaly from Geosat and ERS 1 satellite altimetry. - J. Geophys. Res.B(102): 10039-10054.

SEARLE, R.C., 1989: Location and segmentation of the Cocos-Nazca spreading centre west of 95 degrees W. - Mar. Geophys. Res. 11: 15-26.

SPRECHMANN, P., ASTORGA, A., CALVO, C. \& FERNÁNDEZ, A., 1994: Stratigraphic chart of the sedimentary basins of Costa Rica, Central America. - Profil, 7: 427-433.

SINTON, C.W., DUNCAN, R.A. \& DENYER, P., 1997: Nicoya peninsula, Costa Rica: A single suite of Caribbean oceanic plateau magmas. - J. Geophys. Res. 102 (B7): 15507-15520.
SMITH, W. H. F. \& SANDWELL, D.T., 1997: Global sea floor topography from satellite altimetry and ship depth soundings. Science, 277: 1956-1962.

VON HUENE, R., BIALAS, J., FLUEH, E.R., CROPP, B., CSERNOK, T., FABEL, E., HOFFMANN, J., EMEIS, K., HOLLER, P., JESCHKE, G., LEANDRO-M., C., PERÉZ-FERNANDÉZ, I., CHAVARRIAS. J., FLOREZ-H., A., ESCOBEDO-Z., D., LEÓN, R. \& BARRIOS-L., O., 1995: Morphotectonics of the Pacific convergent margin of Costa Rica. - En: P. MANN (ed.): Geologic and Tectonic Development of the Caribbean plate boundary in southern Central America. - Geol. Soc. Am., Spec. Pap. 295: 291-308.

WERNER, R., HOERNLE, K., VAN DEN BOGAARD, P., RANERO, C., VON HUENE, R., \& KORICH, D., 1999: Drowned 14-m.y.-old Galápagos archipelago off the coast of Costa Rica: Implcations for tectonic and evolutionary models. - Geology, 27: 499-502.

WILSON, D.S. \& HEY, R., 1995: History of rift propagation and magnetization intensity for the Cocos-Nazca spreading center. - J. Geophys. Res. 100(B7): 10041-10056.

WILSON, D.S., 1996: Fastest known spreading on the Miocene Cocos-Pacific plate boundary. - Geophys. Res. Lett. 23: 3003-3006. 